\title{
Developing a Multiplayer Online Learning Environment to Web Support with AJAX3D and Virtual Reality
}

\begin{abstract}
This paper presents Ludos Top - an educational $3 D$ game that use virtual reality techniques, which can support multi-student with a new design model of networking on the web.

The project has actively involved end-users to focus on increase interactivity through the use of versatile system architecture.

We present a quick prototyping of a multi-user virtual world through the employment of Ajax, X3D and Web Services provides an efficient, flexible and robust means for distributed application. Results show improved network capabilities, in terms of interactive, ease of use, enjoyability, playability and usability.
\end{abstract}

Keywords: Ajax3D Distributed Virtual Worlds, Online Games, and Web Games.

\section{INTRODUCTION}

The word games refer to activities of which nature or finality is recreative, diversion and entertainment. Theses activities exists officially since 776 B.C., and they began in Olympia, old Greece, with the Olympic Games. The games perpetuates till nowadays, nevertheless, the way which they are realized and its propose changed.

Research work argues that computer games are an engaging medium for learning since they can stimulate cognitive processes as reading explicit and implicit information, deductive and inductive reasoning, problem solving, and making inferences from information displayed across a number of screens. According to constructivist approach [Von Glaserfeld, 1990], learning depends on the active engagement of the subject that learns and on his ability to construct knowledge and understanding on the basis of interaction with the environment.

The Web environment has matured to support realtime delivery of web-based 3D content to increase interaction and integration with others systems. The Virtual Reality demonstrates the same evolution through of new standards like: X3D, the successor of VMRL standard for Web-based 3D graphics.

The X3D is an open standard file format and API for representing and communicating with Scene Authoring Interface (SAI). A major goal of X3D is to support 3D web applications, in addition to $3 \mathrm{D}$ on networking with portability.

This show news paradigms e.g. integration of X3D with Ajax3D programming model which is the W3C Document Object Model (DOM). The Ajax3D consists of a web-page embedded JavaScript program which allows an architecture asynchronously given flexibility for manipulation.

Through Ajax3D and Web Services on architecture application-to-application communication via network or the web does solve many of the problems, it also creates many new problems. The focus is to support the movement towards an efficient environment that enables the development of multi-user applications, providing functionality with the highest level of compatibility.

This work motivated the development a game with pedagogical objectives. It is called by Blaise Müller of Quatro and particularly useful to test observation and thought abilities.

This paper presents works related. Section Three give a view about background. Section Four provides an overview of our proposed architecture. Section Five provides the case study Ludos Top. Section Six shows system implementation of this technology in a simulation. Section seven provides a conclusion.

\section{RELATED WORKS}

The increasing number of broadband users, and demand for service quality and diversity, especially in the entertainment area, drive to development of the new games like:

Strike Fighter - was developed by Larry Rosenthal of Cube Productions. It has been running a science fiction virtual world/online community called StarbaseC3 for nearly that long. In 2001 were developed the first version of a 3D web game called "Strikefighter," in VRML programming. Last year Strkefighter was updated for X3D and showed new model based on architecture with Ajax3D, the game connected with web server (running PHP and MySQL) that implements a scoreboard. When the game is over, it checks the current score against a database of high scores residing on the server. Strikefighter is similar to the Flash-based mini-games that have proliferated on the web over the last several years.

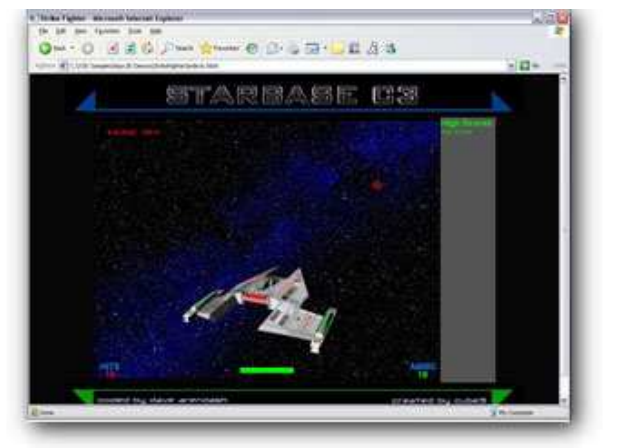

Figure 2: Snapshot from Strile Fighter game in Ajax3D.

The Strike Fighter involved by: JavaScript, XMLHttpRequest, the DOM and SAI running in a web browser-independent fashion that will work with IE, Firefox and others browsers. 
Road Rider - is an interactive first person 3D game, where the player controls a virtual character whose task consists in reaching the site of a rock concert. During her/his trip, the user walks around a city (that is a 3D reconstruction of a portion of the Genoa city center - figure 3). The game plot consists of a number of "missions". Missions involve finding a car, getting money to buy a ticket for the concert, driving the car to visit friends who live in different cities to another, and finally reaching the destination site. Every mission features an increasing level of difficulty. In order to enhance the player's engagement, the game plot is dynamic. All the important game situations are tied to road safety (road-signs, vehicles, roads, cross-roads, pedestrians, etc.). And this is true also for the score rules. Score is a fundamental element of the game, since it provides the main motivation for a user to improve her/his performance. So, the criteria according to which points are assigned are very important because they define what layers' operations, actions and behaviours are positive and what are not relevant or even negative. In Road Rider, the system penalizes hazardous behaviors and rewards safe road-behaviors.

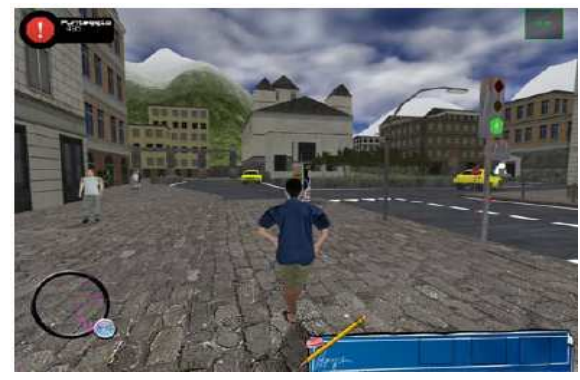

Figure 3: Snapshot from Road Rider.

The game provides a valid support to realize:

- a realistic 3D visualization, which is quite complex to achieve in a networked environment;

- an effective narrative, which is important in order to engage the user in interesting and compelling situations;

- A realistic simulation, which is important to increase the likelihood that the user may transfer in the real-life techniques and skills learned through simulation.

The NICE project - is an effort to build Narrativebased, Immersive, Constructionist/Collaborative Environments for children. Developed at the Interactive Computing Environments Laboratory (ICE) and the Electronic Visualization Laboratory (EVL) of the University of Illinois at Chicago, NICE aims to create a virtual learning environment that is based on current educational theories of constructionism, narrative, and collaboration, while fostering creativity within a motivating and engaging context.

NICE is an outgrowth of two previously designed systems, CALVIN and the Graphical Storywriter.

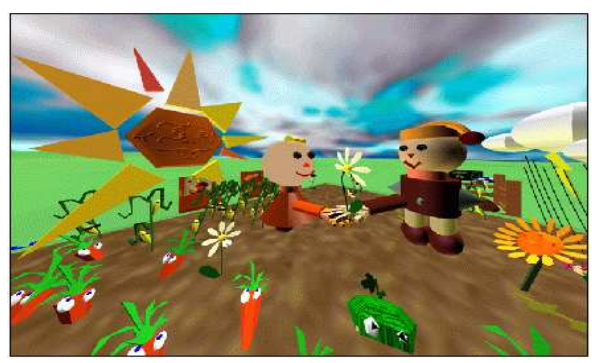

Figure 4: Snapshot from Narrative, Immersive, Constructionist/Collaborative Environments.

Equally important to the construction of one's knowledge is the experience gained by participation in group activity. Collaboration is emphasized in our framework through the combination of collaborative learning across both virtual, as well as physical communities. Collaboration of virtual communities refers to communication and shared experience between children who are geographically separated.

The network component of NICE allows multiple networked participants at different locations to interact with each other and share the same virtual space.

The number of participants is limited only by bandwidth and latency of the network. Multiple distributed NICE applications running on separate VR systems are connected via the central LIFE server across all the separate environments. The communications library uses multicasting to broadcast positional and orientation information about each child's avatar, and uses TCP/IP to broadcast state information between the participants and the behavior system [M. Roussos, A. E. Johnson, J. Leigh, C. R. Barnes, C. A. Vasilakis, and T. G. Moher, 1997].

\section{3. BACKGROUND}

The 3D web environment have begun to demonstrate increased utility which include higher use of participation, scalability and compatibility.

\subsection{Ajax3D}

The technology Ajax has architecture neutral approach due new standard of web-based development. One of the most popular trends in web applications is enriching client functionality through the means of Asynchronous Javascript and XML (Ajax) [Crane, David, Pascaraello, Eric, 2005]. AJAX is a combination of existing technologies rather than a completely new innovation. The primary component is the XMLHTTPRequest (XHR) object that provides for the ability to perform asynchronous communication with a server via Hypertext Transfer Protocol (HTTP). Used in coordination with either Dynamic Hypertext Markup Language (DHTML) or use of HTML FRAMES, the XHR request object allows a high level of performance thus avoiding the call-and response mechanism of earlier web applications.

Replacing the integration of DHTML functionality with the X3D event model, applications have applied 
the use of X3D markup technologies in the context of an AJAX-based application[Parisi, Tony, 2006]. This approach has identified a trend of scripting the 3D scene hierarchy in the same fashion as the Document Object Model (DOM) and handling the events through Javascript. By relying on AJAX-based network calls, a user interacts with backend tiers via a $3 \mathrm{D}$ scene in asynchronous fashion previously provided by employment of HTML 'DIV' tags. This technique has supported a much higher level of interactivity and efficiency in web-based 3D [A. Ostrowski, David, 2007].

\subsection{Multiplayer Worlds}

Recently, the term Post-Nintendo kids have been coined, referring to the fact that today most children have been exposed to 3D computer games. With ease they navigate through virtual environments interpreting audio-visual hints as they go. Just, as WIMP (windows, menus, pull-downs) metaphor rules the design of graphical user interface (GUI) of today's software and replaced the command line control, 3D environment and 3D navigation with new GUI metaphors for operation systems and desktop applications.

Multiplayer worlds are a new way for people to collaborate. Clarker-Willson (S. Clarker-Willson., 1998.) suggest that virtual environment designers should apply principles that made even early games so appealing: third person view, discovery and exploration, player control, maps, landmarks, closed environments (limited space), complexity management (reduced number of active objects) and constant positive feedback.

Platforms of increasing importance for multiplayer worlds are game console.

\subsection{Trends in Multi-user and Gaming Applications}

The multiplayer games are notoriously difficult to implement correctly of effectively because they are multiple traditional types of software rolled into a single application, like: Graphical applications, Distributed Systems and Interactive applications.

The multiplayer game industry has influenced applications including simulation, education and training [Jain, Sanjay, McLean, Charles R., 2005].

In principle, developers have relied on $\mathrm{C}$ and $\mathrm{C}++$ exclusively for gaming development [Davison, Andrew, 2005]. While providing a tight coupling with operating systems, this approach is slowly changing towards development in languages that are more portable and provide for faster development cycles. Nowadays, already exist game's architecture implemented for compatibility and ease of development, for WWW through of new technologies shows next-generation gaming architecture with rapid development.

\section{4. OVERVIEW OF ARCHITECTURE}

The architecture shows the integration between Ajax, X3D and Web Services that introduces a new solution for multi-user gaming system. This solution provides some advantages like: scalability, security and interactive responsiveness.

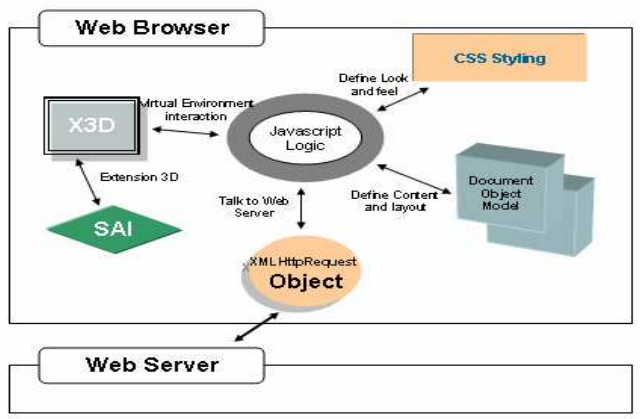

Figure 4: Architecture based X3D and Ajax

AJAX have found acceptance across a number of web based games starting from board based games such as WEBGOGGLE and working towards 3D interactive games including Strike Fighter. While some of the architectures surveyed at this writing maintain similar qualities to our application, direct comparisons are difficult to perform. Advantages of our approach include support of web services, a portable solution that could be implemented across a number of architectures. The architecture with Ajax communicating with a web server without refreshing the whole page.

The main goal this project is propose a open architecture distributed portable across platforms, providing support for communication to non X3D applications, allows the routing of events over a network using the same routing, high efficiency transfer of data or node, secure communication and support the main Web/internet standards.

\subsection{Communication Architecture}

The primary advantages of using a distributed communication are scalability, responsiveness and resiliency. Distributed communication allows players to send messages directly to each other, creating a more responsive game. The key challenges in developing communications components for a distributed architecture are a consistency, event ordering, interactive responsiveness, security and scalability.

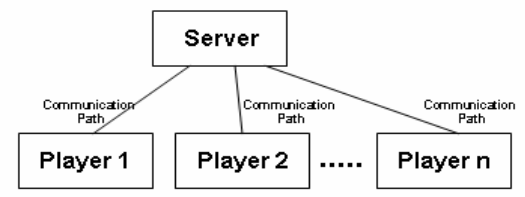

Figure 6: Multi-User client/server (logical architecture) 


\section{LUDOS TOP}

The system proposed based in game Quarto, created by Frenchman Blaise Müller in 1985. The sixteen game pieces show all combinations of size (short or tall), shade (light or dark), solidity (shell or filled), and shape (circle or square). Two players take turns placing pieces on a four by four board and the object is to get four in a line with the same characteristic - all short, for example. Only one piece can go in a cell and, once placed, the pieces stay put. Blaise Müller's brilliant twist is that you choose the piece that your opponent must place and they return the favor after placing it.

\subsection{Functioning of the game}

As the objective of the game is establish a line of four pieces, with at least one common characteristic on the board. The line of pieces may be across the board, up and down, or along a diagonal.

Game sequence when the first player selects one of the 16 pieces and gives it to his opponent. That player places the piece on any square on the board and chooses one of the 15 pieces remaining and gives it to his one. The game is won by the first player to call "QUARTO!"

\subsection{Aspect Pedagogical}

Nowadays, an increasing demand for greater interactivity to be built into learning materials. There is a clear need to offer a variety of different knowledge presentations and to create opportunities to apply the knowledge within the virtual world, thus supporting and facilitating the learning process. To achieve that, it is necessary to provide a complex level of interactivity that stimulates users' engagement, apply different interactivity concepts as object, linear, construct or hyperlinked interactivity, and non-immersive contextual interactivity as well as immersive virtual interactivity.

The technical advantages are employing interactive through of Ajaxian techniques that provides better enjoyability, playability and usability

\section{SYSTEM IMPLEMENTATION}

The implementation of the system denominates Ludos Top which introduces the game Quatro and some systems like: Ajax Techniques, Flex Flux plug-in 3D, Internet Explorer browser and Tomcat.

\subsection{Ludos Top Web User Interface}

The Ludos Top interface is a HTML programmed using JavaScript, Ajax and X3D. The communication between X3D and HTML page is through the X3D Script SAI, after this, the parameters are forwarded outside world for Web Services using Ajax request with XMLHttpRequest.

\section{CONCLUSION}

In this paper presents the possibilities of fully distributing multi-user games online through of standards such as X3D have made it possible deploy rich $3 \mathrm{D}$ content in real time over the Internet.

At the same time, Ajax has emerged as a worldwide phenomenon and an interest of new application development. By bringing these two technologies together, Ajax3D promises to be good open platform for creating a next-generation 3D web experience.

With Ajax3D, immersive virtual worlds can be deployed within a web browser, integrated with pages with more interaction, compatibility and can communicate with standard web servers using XML and Ajax technologies, enabling networking on web.

The Ludos Top is in its infancy. It will need to be actively developed. Ludos Top can become an example of Educational Virtual World on World Wide Web for help research and development personal.

\section{ACKNOWLEDGEMENTS}

The authors would like to thanks the Luciano and Marlene Roque at the University Federal of Uberlândia for supporting the establishment of the Computer Graphics lab.

\section{REFERENCES}

A. OstrowsKi, DAVID, 2007. A Web-Based 3D Gaming Style Multi-User Simulation Architecture. Ford Research and Advance Engineering.

LeIGH, J. AND Johnson, A. E.1996a. Supporting Transcontinental Collaborative Work in Persistent Virtual Environments. In IEEE Computer Graphics and Applications. July 1996, pp. 47-51.

M. Roussos, A. E. Johnson, J. Leigh, C. R. Barnes, C. A. VASILAKIS, AND T. G. MoHER, 1997. The NICE project: Narrative, Immersive, Constructionist/Collaborative Environments for Learning in Virtual Reality. In IEEE Computer Graphics and Applications.

Steiner, K. E. AND Moher, T. G. 1994. Scaffolding Story Construction with Interactive Multimedia. In The Journal of Educational Multimedia and Hypermedia, pp.173-196.

S. Clarker-Willson., 1998. Applying Game Design to Virtual Environments. In C. Dodsworth (editor), Digital Illusion: Entertaining the Future with High Technology. ACM Press.

Jain, SAnjay, McLean, Charles R., 2005. Integrated Simulation and Gaming Architecture for Incident Management Training. PROCEEDINGS OF THE 2005 Winter Simulation Conference.

E. VON GlaserSFeld, 1990. "Constructivist Views on the Teaching and Learning of Mathematics". Journal for Research in Mathematics Education. Monograph, Vol. 4, pp. 19-29+195-210.

PARISI, TONY, 2006. http://www.ajax3d.org/ whitepaper. 